\title{
Reduction of multi-compartmental biophysical models by incremental, automated retuning of their parameters and synaptic weights
}

\author{
Thomas G Close ${ }^{1 *}$, Ben Torben-Nielsen ${ }^{1}$, Erik De Schutter ${ }^{1,2}$ \\ From The Twenty Third Annual Computational Neuroscience Meeting: CNS*2014 \\ Québec City, Canada. 26-31 July 2014
}

When simulating large networks of neuronal models with conductance-based membrane dynamics, neuronal models with large numbers of compartments are often not practical due to the computational resources required. In this case, a common approach is to seek a model with fewer compartments that approximates the behaviour of the full compartmental model $[1,2]$. While analytical techniques to reduce multi-compartmental models consisting of passive, quasi-active or restricted classes of active models exist [3-5], in general, the reduction of active models requires parameter retuning in order to retain functionally relevant properties of the model. We propose an incremental, automated parameter retuning approach to reduce the number of compartments, which avoids labor-intensive manual retuning and ensures key functional properties of the model are maintained.

Starting from the full compartmental model, the number of compartments is incrementally reduced by merging compartment subsets into single, electronically equivalent compartments. After each compartment reduction phase, all parameters of the model, including synaptic weights, are locally optimised to best match the dynamics and post-synaptic potentials of the full compartmental model using multiple objectives, including phase-plane trajectory histograms of membrane voltage and internal states $[6,7]$. The successive local optimisations during the incremental reduction in the number of compartments ensures the model parameters do not transition to qualitatively distinct regions of the parameter space. The multi-compartmental models are iteratively reduced in this manner until key functional properties can no longer be maintained within specified tolerances.

The proposed algorithm is demonstrated on multicompartmental models of neurons in the cerebellum.

\section{Authors' details}

${ }^{1}$ Computational Neuroscience Unit, Okinawa Institute of Science and Technology, Okinawa, Japan. ${ }^{2}$ University of Antwerp, Antwerp, Belgium.

Published: 21 July 2014

\section{References}

1. Bush PC, Sejnowski TJ: Reduced compartmental models of neocortical pyramidal cells. J. Neurosci. Meth 1993, 46:159-166.

2. Davison AP, Feng J, Brown D: A reduced compartmental model of the mitral cell for use in network models of the olfactory bulb. Brain Res Bull 2000, 51(5):393-9.

3. Rall W: Branching dendritic trees and motoneuron membrane resistivity. Exp Neurol 1959, 1(5):491-527.

4. Ohme M, Schierwagen A: An equivalent cable model for neuronal trees with active membrane. Biol Cybern 1998, 78(3):227-43.

5. Hedrick KR, Cox SJ: Structure-preserving model reduction of passive and quasi-active neurons. J Comput Neurosci 2013, 34(1):1-26.

6. Druckmann S, Banitt Y, Gidon A, Schürmann F, Markram H, Segev I: A novel multiple objective optimization framework for constraining conductance-based neuron models by experimental data. FNINS 2007, 1(1):7-18.

7. Van Geit W, Achard P, De Schutter E: Neurofitter: a parameter tuning package for a wide range of electrophysiological neuron models. Frontiers in Neuroinformatics 2007, 1:1-18.

doi:10.1186/1471-2202-15-S1-P178

Cite this article as: Close et al: Reduction of multi-compartmental biophysical models by incremental, automated retuning of their parameters and synaptic weights. BMC Neuroscience 2014 15(Suppl 1): P178.

\footnotetext{
* Correspondence: tclose@oist.jp

${ }^{1}$ Computational Neuroscience Unit, Okinawa Institute of Science and

Technology, Okinawa, Japan

Full list of author information is available at the end of the article
} 